\title{
Spin precession and modulation in ballistic cylindrical nanowires due to the Rashba effect
}

\author{
A. Bringer ${ }^{1,3}$ and Th. Schäpers ${ }^{2,3, *}$ \\ ${ }^{1}$ Peter Grünberg Institut (PGI-1), Forschungszentrum Jülich GmbH, D-52425 Jülich, Germany \\ ${ }^{2}$ Peter Grünberg Institut (PGI-9), Forschungszentrum Jülich GmbH, D-52425 Jülich, Germany \\ ${ }^{3}$ JARA-Fundamentals of Future Information Technology, Jülich-Aachen Research Alliance
}

(Received 6 November 2010; revised manuscript received 12 January 2011; published 4 March 2011)

\begin{abstract}
The spin precession in a cylindrical semiconductor nanowire due to Rashba spin-orbit coupling has been investigated theoretically using an InAs nanowire containing a surface two-dimensional electron gas as a model. The eigenstates, energy-momentum dispersion, and the energy-magnetic field dispersion relation are determined by solving the Schrödinger equation in a cylindrical symmetry. The combination of states with the same total angular momentum but opposite spin orientation results in a periodic modulation of the axial spin component along the axis of the wire. Spin-precession about the wires axis is achieved by interference of two states with different total angular momentum. Because a superposition state with exact opposite spin precession exists at zero magnetic field, an oscillation of the spin orientation can be obtained. If an axially oriented magnetic field is applied, the spin gains an additional precessing component.
\end{abstract}

DOI: 10.1103/PhysRevB.83.115305

PACS number(s): 72.25.-b, 85.75.Hh, 73.23.-b

\section{INTRODUCTION}

Semiconductor nanowires are almost-ideal objects for studying quantum effects and electron interference phenomena. The use of the bottom-up approach for nanowire growth simplifies the preparation substantially and allows us to create novel confinement schemes, such as axial and radial heterostructures. ${ }^{1,2}$ The high surface-to-volume ratio of nanowires means that surface properties are crucial for discussions of transport properties, so that low-band-gap semiconductors, for example, InAs, InN, or InSb, are particularly interesting. In these systems, the Fermi level at the surface is pinned inside the conduction band, ${ }^{3}$ and an accumulation layer is formed. This guarantees that the conductance is sufficiently large even at low nanowire radius. The presence of the surface accumulation layer means that a tubular conducting channel is formed, and this shape of the conductor has important implications for the magnetoconductance of the nanowires. An example is the theoretical prediction and experimental confirmation of flux-periodic oscillations in nanowires with a magnetic field applied along the wire axis. ${ }^{4,5}$ The electronic states of a cylindrical two-dimensional electron gas (2DEG) in a transverse magnetic field were calculated by Ferrari et al., ${ }^{6,7}$ while Magarill et al. ${ }^{8,9}$ discussed the kinetics of electrons in a tubular conductor.

Many concepts have been developed for planar semiconductor layer systems that make use of the spin degrees of freedom for device structures. The best-known example is the spin-field-effect transistor, ${ }^{10-12}$ which uses the gate-controlled spin precession induced by the Rashba effect. ${ }^{13-15}$ The Rashba spin-orbit coupling originates from a macroscopic electric field in an asymmetric quantum well. ${ }^{16}$ Meanwhile, research activities have been extended to planar quasi-one-dimensional structures, which promise superior spin control. ${ }^{17-19}$ The energy spectrum and spin precession in these structures are governed by the interplay between confinement and energy splitting due to spin-orbit coupling. ${ }^{20,21}$ Only a few theoretical investigations have dealt with the effect of spin-orbit coupling in cylindrical conductors on electronic states and on quantum transport. ${ }^{4,9,22,23}$ The spin dynamics in curved 2DEGs was discussed by Trushin and Schliemann, ${ }^{24}$ while the weak antilocalization effect in cylindrical wires was studied by Wenk and Kettemann. ${ }^{25}$ The presence of spin-orbit coupling was confirmed for InN and InAs semiconductor nanowires by measuring the weak antilocalization effect. ${ }^{26-30}$

The various possibilities of spin control in 2DEGs and planar wire structures opened up by the Rashba effect have inspired us to analyze theoretically the spin dynamics in tubular conductors. We have used a cylindrical InAs nanowire with a surface 2DEG as a model system, but our findings also apply to other systems, for example, $\mathrm{InN}$ or InSb nanowires. In Sec. II we analyze the electronic states, focusing on spin properties, and we discuss the conditions under which a spin precession can be observed in tubular nanowires at zero magnetic field (Sec. III) and in an axial magnetic field (Sec. IV). In Sec. V, we comment on the suitability of tubular conductors for spin electronic devices.

\section{ELECTRONS IN CYLINDRICAL WIRES}

Electrons confined in a cylinder move along the axis with a linear momentum $\hbar k$ ( $k$ real) and around the axis with an angular momentum $\hbar l$ ( $l$ integer). As long as the translational and rotational symmetries of the cylinder are not perturbed, these momenta are conserved quantities. The wave function of an electron

$$
\psi=\exp (\imath k z) \exp (\imath l \phi) f(r)
$$

is a product of exponential functions in $z, \phi$, the coordinate along the axis and the azimuthal angle around the axis, respectively, and a radial distribution function $f(r)$. The distribution is determined by internal forces produced by the cylinder material. In our case, we took a planar 2DEG at the surface of InAs as a reference, ${ }^{31,32}$ that is, assuming a surface state charge density of $N_{S}=1.27 \times 10^{11} \mathrm{~cm}^{-2}$, a background $p$ doping of $n_{d}=2.8 \times 10^{17} \mathrm{~cm}^{-3}$, and an effective electron mass of $m^{*}=0.026 m_{e}$. The calculations were done for a cylinder radius $r_{0}=50 \mathrm{~nm}$. A schematic of the nanowire is depicted in Fig. 1 (upper inset). Electrons of 


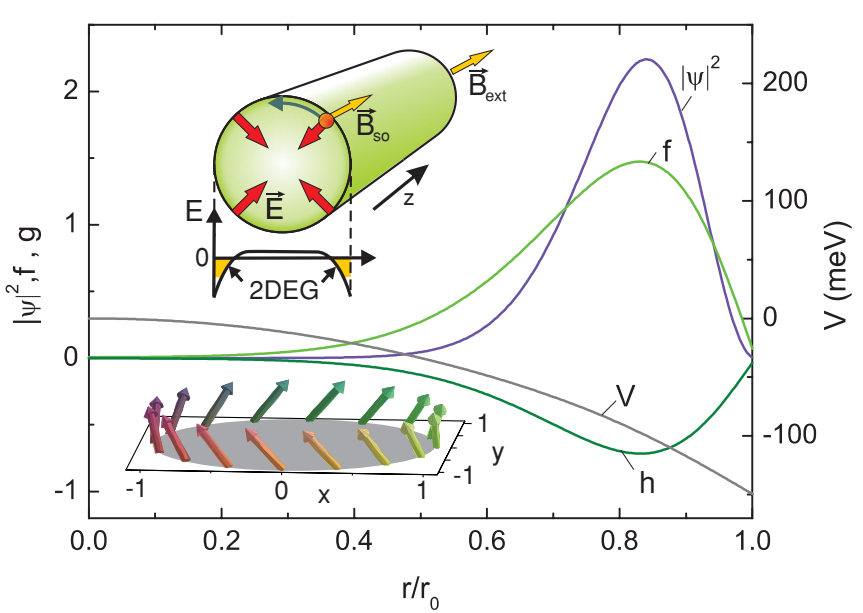

FIG. 1. (Color online) Squared amplitude of the wave function $|\psi|^{2}$, the spinor components $f$ and $h$, and the potential profile $V$ as a function of the normalized radius $r / r_{0}$. Upper inset: Schematic of the nanowires, including the relevant electric and magnetic fields. Lower inset: Spin orientation along the circumference for $j=1 / 2$.

atoms at the surface may find energetically more favorable states in the conduction band. Due to the Coulomb attraction between the electrons and the ions remaining at the surface, the electrons get trapped in a layer close to the surface, forming a 2DEG. ${ }^{33}$ The potential $V$ resulting from the charge density of occupied electron states $\psi_{l, \sigma, k}$, of ions at the surface and of dopants $\rho_{\mathrm{BG}}$,

$$
\rho=\left(e \sum_{l, \sigma, k}^{\mathrm{occ}}\left|\psi_{l, \sigma, k}\right|^{2}+\rho_{\mathrm{BG}}\right) / \epsilon_{r},
$$

is shown in Fig. 1. $e$ is the elementary charge, and $\sigma$ the spin index. $\epsilon_{r}=14.6$ is the bulk dielectric constant of InAs. ${ }^{34}$ It takes the polarization charges of the medium into account. The potential profile is determined by Poisson's equation, which is solved in cylindrical symmetry analytically,

$$
V=4 \pi \epsilon_{0} e \int_{0}^{r} r^{\prime} d r^{\prime} \rho\left(r^{\prime}\right) \ln \frac{r^{\prime}}{r} .
$$

Equations (1) and (2) are solved self-consistently. Starting from the potential of a homogeneous distribution of electrons in the cylinder, the distribution is recalculated using the Schrödinger equation given below [see Eq. (5)] and Eq. (1). The iteration procedure converges monotonically. We assumed an interface barrier of infinite height.

Due to the electric field $\overrightarrow{\mathcal{E}}=-\nabla V / e$ across the surface of the cylinder, the spin $\vec{\sigma}$ of the electron is coupled to its orbital motion

$$
\begin{aligned}
\mathcal{H}_{\text {SO }}= & \vec{\sigma} \cdot[\vec{p} \times e \overrightarrow{\mathcal{E}}] \frac{\gamma}{\hbar} \\
= & \gamma V^{\prime}\left[\left(\begin{array}{cc}
0 & \iota e^{-\imath \phi} \\
-\imath e^{\imath \phi} & 0
\end{array}\right) \frac{\partial}{\imath \partial z}\right. \\
& \left.+\left(\begin{array}{cc}
1 & 0 \\
0 & -1
\end{array}\right) \frac{\partial}{r \iota \partial \phi}\right] .
\end{aligned}
$$

The coupling strength $\gamma$ is determined by the band structure of the cylinder material $\left(1.17 \mathrm{~nm}^{2}\right.$ for InAs). ${ }^{34}$ The second part of Eq. (3) expresses $\mathcal{H}_{\mathrm{SO}}$ in terms of Pauli matrices for $\sigma_{x, y, z}$ acting on a two-component (spinor) wave function $\left(\psi_{\uparrow}, \psi_{\downarrow}\right)$. The off-diagonal terms in $\mathcal{H}_{\text {SO }}$ raise(lower) the value of the orbital angular momentum $\mathcal{L}_{z}$ of $\psi_{\uparrow}\left(\psi_{\downarrow}\right)$ by $\hbar$. The stationary states are eigenstates of the total angular momentum $\mathcal{J}_{z}=\mathcal{L}_{z}+\mathcal{S}_{z}\left(\mathcal{S}_{z}=\hbar \sigma_{z} / 2\right)$, with eigenvalues $j=l \pm 1 / 2$. The spinor is of the form

$$
\left(\begin{array}{c}
\psi_{\uparrow} \\
\psi_{\downarrow}
\end{array}\right)=e^{\imath k z} e^{\imath l \phi}\left(\begin{array}{c}
f(r) \\
l e^{\imath \phi} h(r)
\end{array}\right),
$$

where $f, h$ are real functions and solve the differential equations

$$
\begin{aligned}
& -\frac{\hbar^{2}}{2 m^{*}}\left(f^{\prime \prime}+\frac{1}{r} f^{\prime}\right)+\left(\hat{V}_{l,+}-\hat{\epsilon}\right) f=k \gamma V^{\prime} h, \\
& -\frac{\hbar^{2}}{2 m^{*}}\left(h^{\prime \prime}+\frac{1}{r} h^{\prime}\right)+\left(\hat{V}_{l+1,-}-\hat{\epsilon}\right) h=k \gamma V^{\prime} f .
\end{aligned}
$$

Here, $\hat{V}_{l, \pm}=(\hbar l)^{2} /\left(2 m^{*} r^{2}\right)+V \pm \gamma V^{\prime} l / r$ contains the contributions of the centrifugal force and the diagonal spin-orbit term, $\hat{\epsilon}=\epsilon-(\hbar k)^{2} /\left(2 m^{*}\right)$, is the energy without the axial kinetic energy. At the wire boundary we assumed a barrier of infinite height. ${ }^{35}$ The influence of an external magnetic field $B$ is not included yet.

In Fig. 2 the energy $\hat{\epsilon}$ is plotted for several $j$ bands at $B=0$. The parabola indicates the axial kinetic energy left out. It crosses the bands at the Fermi momentum $k_{F}$; that is, states with energy below the parabola are occupied. At $k=0$ the coupling between $l$ and $l+1$ vanishes [cf. Eq. (3)]. Classification with respect to $l$ is possible. The splitting between the second and the third bands $(l= \pm 1)$ is caused by the diagonal part of $\mathcal{H}_{\text {SO }}$ and increases proportional to $l$ for the higher states. Due to the mirror symmetry $z \leftrightarrow-z$ states with angular momentum and spin reversed have the same energy. Therefore, all bands are twofold degenerate.

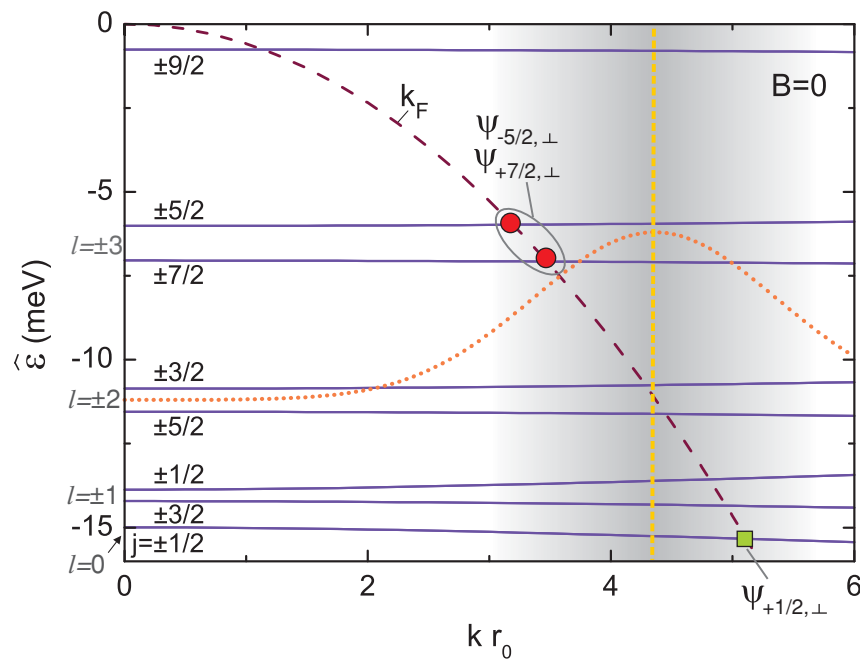

FIG. 2. (Color online) Energy vs. $k$ dispersion at $B=0$. The dashed line indicates the axial kinetic energy left out, which crosses the bands at $k_{F}$. The pair of circles represents states forming the superposition states $\psi_{-5 / 2, \perp}$ and $\psi_{+7 / 2, \perp}$, while the square indicates the state $\psi_{+1 / 2, \perp}$. The dotted line illustrates the Gaussian wave packet of width $\delta k=1 / r_{0}$. 


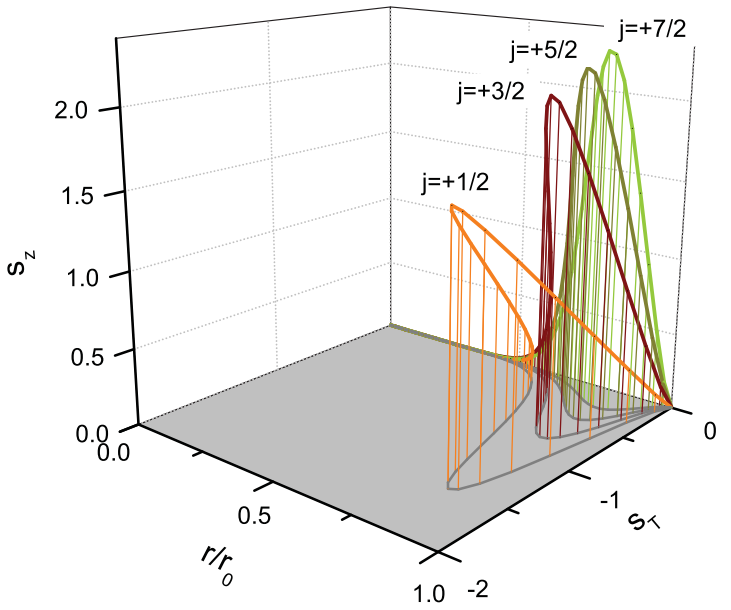

FIG. 3. (Color online) Spin density $\left(s_{T}, s_{z}\right)$ of the lower energy states for total angular momenta $j=1 / 2,3 / 2,5 / 2$, and $7 / 2$. The spin is oriented only tangentially and along the $z$ axis.

The solution ( $f, h)$ of Eq. (5) for $j=1 / 2$ at $k_{F}$ is shown in Fig. 1. The spin-orbit coupling increases linearly with $k$; that is, at $k_{F}$ with $l=0$ there is the strongest spin-orbit coupling. The spin density attains a sizable tangential component:

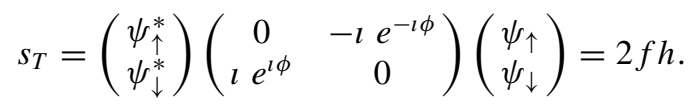

The component along the wire axis is

$$
s_{z}=\left(\begin{array}{c}
\psi_{\uparrow}^{*} \\
\psi_{\downarrow}^{*}
\end{array}\right)\left(\begin{array}{cc}
1 & 0 \\
0 & -1
\end{array}\right)\left(\begin{array}{l}
\psi_{\uparrow} \\
\psi_{\downarrow}
\end{array}\right)=f^{2}-h^{2} .
$$

The radial component is 0 . The spin orientation around the cylinder for $j=1 / 2$ is illustrated in Fig. 1 (lower inset). According to Eq. (6) the spin turns to the axial direction. This is shown for different values of $j$ in the plot of the spin densities $s_{T}$ and $s_{z}$ in Fig. 3. As shown there, the spin is oriented exclusively tangentially and along the axial direction. When averaged over the cylinder plane $\langle\cdots\rangle$ for each state $\psi_{j}$ the spin components $\left\langle\sigma_{x}\right\rangle$ and $\left\langle\sigma_{y}\right\rangle$ are 0 , while a finite contribution $\left\langle\sigma_{z}\right\rangle$ remains along the $z$ direction.

\section{SUPERPOSITION STATES AND SPIN PRECESSION}

For each $k$ and $j$ there are two solutions of Eq. (5), $\psi_{j, \pm}$. The $(+)$ state originates from $\left(\psi_{l}, 0\right)$, and the $(-)$ state from $\left(0, \psi_{l+1}\right)$; the solutions are at $k=0$, with $j=l+1 / 2$. They are orthogonal to each other and have opposite spin direction $( \pm)$. They have different energies $\hat{\epsilon}$ and therefore different $k_{F}$. Their superposition $\psi_{j, \|}=\left(\psi_{j,+}+\psi_{j,-}\right) / \sqrt{2}$ yields

$$
\begin{aligned}
\left\langle\sigma_{z}\right\rangle_{\|}= & \left(\left\langle f_{j,+}^{2}-h_{j,+}^{2}\right\rangle+\left\langle f_{j,-}^{2}-h_{j,-}^{2}\right\rangle\right) / 2 \\
& +\left\langle f_{j,+} f_{j,-}-h_{j,+} h_{j,-}\right\rangle \cos \left(k_{F,+}-k_{F,-}\right) z .
\end{aligned}
$$

The contributions of the basis states $( \pm)$ almost cancel each other and are neglected henceforth. The interference between the states is constructive due to orthogonality and leads to

$$
\left\langle\sigma_{z}\right\rangle_{\|} \approx 2\left\langle f_{j,+} f_{j,-}\right\rangle \cos \left(k_{F,+}-k_{F,-}\right) z,
$$

an oscillation of the average spin along the cylinder axis with a wavelength $\lambda_{\|}=2 \pi /\left|k_{F,+}-k_{F,-}\right|$. The spin components $\left\langle\sigma_{x}\right\rangle_{\|},\left\langle\sigma_{y}\right\rangle_{\|}$in the cylinder plane are both 0 .

Superpositions of eigenstates with different $j$ values form states with a nonzero average spin component in the cylinder plane, for example, $\psi_{j, \perp}=\left(\psi_{j,+}+\psi_{j-1,-}\right) / \sqrt{2}$. As one can easily retrace, these states originate from states with the same angular momentum $l$. The interference term gives the only $\phi$-independent contribution to the densities of $\sigma_{x}, \sigma_{y}$. With

$$
\begin{gathered}
\psi_{j,+}=\exp \left(\imath z k_{F,+}\right) \exp (\iota l \phi)\left(\begin{array}{c}
f(r) \\
\iota e^{\imath \phi} h(r)
\end{array}\right), \\
\psi_{j-1,-}=\exp \left(\iota z \tilde{k}_{F,-}\right) \exp [\imath(l-1) \phi]\left(\begin{array}{c}
\tilde{f}(r) \\
\iota e^{\imath \phi} \tilde{h}(r)
\end{array}\right),
\end{gathered}
$$

the averages are

$$
\begin{aligned}
& \left\langle\sigma_{x}\right\rangle_{\perp}=\langle h \tilde{f}\rangle \sin \left(k_{F,+}-\tilde{k}_{F,-}\right) z, \\
& \left\langle\sigma_{y}\right\rangle_{\perp}=\langle h \tilde{f}\rangle \cos \left(k_{F,+}-\tilde{k}_{F,-}\right) z .
\end{aligned}
$$

The $\left\langle\sigma_{z}\right\rangle_{\perp}$ contribution is small and does not depend on $z$. In particular, for the superposition $\psi_{1 / 2, \perp}$ of the lowest two states, $\left\langle\sigma_{z}\right\rangle_{\perp}$ is 0 .

For $\psi_{-5 / 2, \perp}$, the superposition of $\psi_{-5 / 2,+}$ and $\psi_{-7 / 2,-}$, the spin precesses counterclockwise in the cylinder plane along the cylinder axis, as illustrated in Fig. 4(a). There, we assumed an initial spin orientation along the $-y$ direction, which in practice can be realized by spin injection from a spin-polarized electrode. For $\psi_{+7 / 2, \perp}$ constituted of the opposite states $\psi_{+7 / 2,+}$ and $\psi_{+5 / 2,-}$ the spin precession is clockwise. Both precessions have the same period of $\lambda_{\perp}=2 \pi /\left|k_{F,+}-\tilde{k}_{F,-}\right|$. Their energy is degenerate. Due to their exactly inverse precession sense, the combination of these states results in an oscillatory behavior of the net spin orientation, as depicted in Fig. 4(b). For an initial spin orientation along the $-y$ direction the spin oscillates in the $y z$ plane. Superposition of the respective opposite states restores the left-right symmetry and eliminates spin precession. The oscillation period $\lambda_{\perp}$ of $\psi_{j, \perp}$ depends on $j$. For smaller $|j|$, for example, $\psi_{-3 / 2, \perp}$, the corresponding difference in $k_{F,+}$ and $\tilde{k}_{F,-}$ becomes smaller so that the period $\lambda_{\perp}$ is enlarged, as one can infer from comparison of Fig. 4(b) to Fig. 4(c). The superposition state $\psi_{+1 / 2, \perp}$ constituted of the two lowest- lying energy states $\psi_{ \pm 1 / 2, \pm}$ (cf. Fig. 2; square) shows no precession at all, because here $k_{F,+}$ and $\tilde{k}_{F,-}$ are identical. Figure 4(d) shows the spin variation for a Gaussian wave packet of width $\delta k=1 / r_{0}$ centered between the $k_{F}$ values of the states $\psi_{-3 / 2, \perp}$ and $\psi_{+5 / 2, \perp}$. In position space this corresponds to a distribution of width $2 r_{0}$. The oscillation deviates from a purely harmonic oscillation, as shown in Fig. 4(c), due to the contributions of the other states at the Fermi energy. This effect is also increasing with decreasing $|j|$ when the $k_{F}$ values get closer to each other.

The electron spin is usually injected from a spin-polarized electrode in all states at the Fermi energy $E_{F}$ having the correct spin direction. Thus, if only the direction of the spin is fixed by the electrode, all states are likely to transport electrons through the cylinder and a definite precession will not be observed. The total spin will only vary in the plane that is defined by the initial spin orientation and the $z$ axis, similar to 

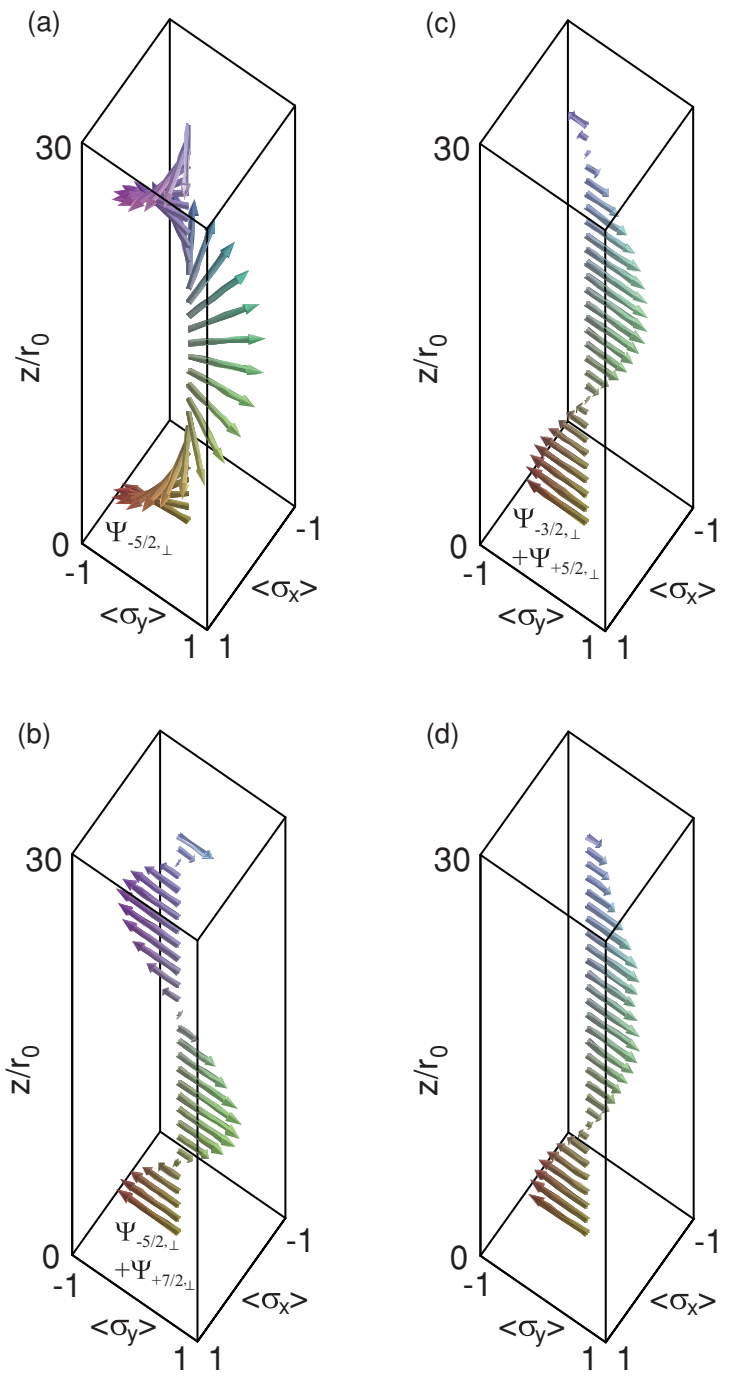

FIG. 4. (Color online) (a) Counterclockwise spin precession of electrons in the superposition state $\psi_{-5 / 2, \perp}$ at the Fermi energy constituted of the states $\psi_{-5 / 2,+}$ and $\psi_{-7 / 2,-}$ for a propagation along the wire axis from $z / r_{0}=0$ to 30 . (b) Spin orientation of the sum of the contribution shown in (a) and the corresponding clockwise contribution $\psi_{+7 / 2, \perp}$, being a superposition of $\psi_{+7 / 2,+}$ and $\psi_{+5 / 2,-}$. (c) Spin oscillations resulting from the combinations of the two lower energy superposition states, $\psi_{-3 / 2, \perp}$ and $\psi_{+5 / 2, \perp}$. (d) Spin variation for a Gaussian wave packet of width $1 / r_{0}$ centered between the $k_{F}$ values of the states $\psi_{-3 / 2, \perp}$ and $\psi_{+5 / 2, \perp}$ (cf. Fig. 2).

the situation illustrated in Fig. 4. To observe spin precession about the cylinder axis, a selection mechanism that breaks the left-right symmetry of the system must be adopted. As discussed in Sec. IV, this is achieved by applying a longitudinal magnetic field $\overrightarrow{\mathcal{B}}=(0,0, B)$.

\section{SPIN PRECESSION IN A MAGNETIC FIELD}

The vector potential $\overrightarrow{\mathcal{A}}=(-B y / 2, B x / 2,0)$ of a longitudinal magnetic field introduces a paramagnetic (Zeeman) and diamagnetic (Landau) term into Eq. (5). $\hat{V}_{l, \pm}$ is extended to

$$
\tilde{V}_{l, \pm}=\hat{V}_{l, \pm}+\frac{\hbar e}{2 m^{*}} B\left(l \pm \frac{g m^{*}}{2 m_{e}}\right)+\frac{e^{2} B^{2}}{8 m^{*}} r^{2}
$$

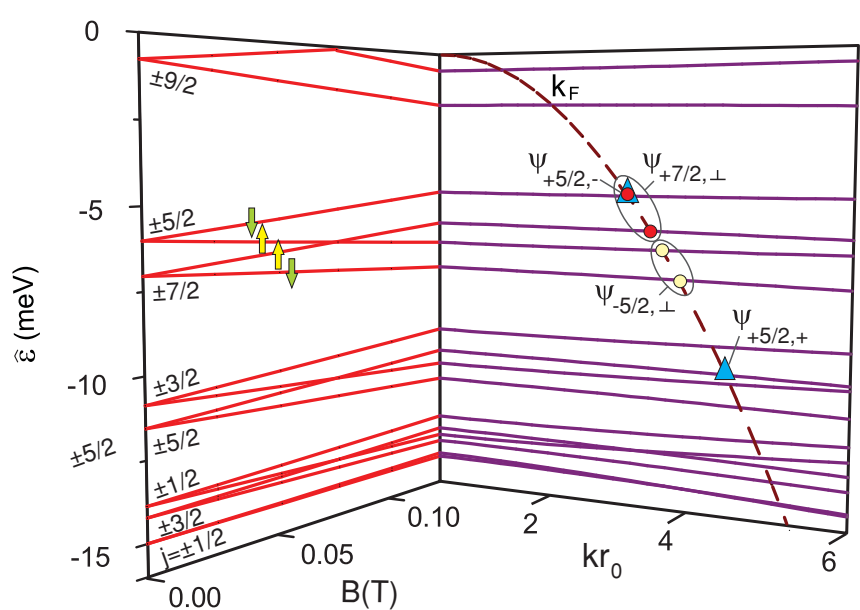

FIG. 5. (Color online) Energy vs. $B$ dispersion (left) at $k=0$ and energy vs. $k$ dispersion (right) at $B=0.13 \mathrm{~T}$. The dashed line indicates the axial kinetic energy left out, which crosses the bands at $k_{F}$. The pairs of circles indicate the states forming the superposition states $\psi_{-5 / 2, \perp}$ and $\psi_{+7 / 2, \perp}$ at $k_{F}$ with a net spin in the cylinder plane. The two states $\psi_{+5 / 2,+}$ and $\psi_{+5 / 2,-}$, with $j=+5 / 2$, are shown by triangles.

with $g$ the gyromagnetic factor of the electron spin $(-14.9$ for InAs). ${ }^{34}$ The paramagnetic (second) term in $\tilde{V}_{l, \pm}$ raises $\hat{\epsilon}$ for states with $j$ (or $l$ ) $>0$ and lowers $\hat{\epsilon}$ for states with $j$ (or $l)<0$. The energy difference increases $\propto l B$ for $B \ll$ $l \hbar /\left(e r_{0}^{2}\right)$ (cf. Fig. 5). For larger $B, \hat{\epsilon}$ increases $\propto B^{2}$ due to the diamagnetic (third) term. In the linear range the influence of the $r$ dependence of the third term is negligible. The densities do not change significantly.

The main effect of $B$ is the energetic separation of the $\pm j$ states. This opens possibilities of observing spin dynamics in electronic transport, which is demonstrated in the following at $B=0.13$ T. Figure 5 shows the $B$ dependence at $k=0$ up to $B=0.13 \mathrm{~T}$ and the $k$ dependence at $B=0.13 \mathrm{~T}$ of $\hat{\epsilon}$ for states from $j= \pm 1 / 2$ to $\pm 9 / 2$. Again, the parabola marks the Fermi edge. Superpositions with spin in the cylinder plane according to Eq. (9), $\psi_{j, \perp}$, are marked as pairs in Fig. 5. The lower pair corresponds to $\psi_{-5 / 2, \perp}$ depicted in Fig. 4(a). As illustrated in Fig. 6(a), it shows the same counterclockwise precession. In contrast to the zero-field case, now the superposition state $\psi_{+7 / 2, \perp}$ has a larger $k_{F}$ difference, that is, a shorter precession length [cf. Fig. 6(b)]. Consequently, the precessions of $\psi_{-5 / 2, \perp}$ and $\psi_{+7 / 2, \perp}$ are not exactly opposite. In contrast to the case at $B=0$, the spin still rotates following the state with the faster precession, when both states are superposed. This is illustrated in Fig. 6(c), where one finds that, in addition to the oscillation of the spin amplitude, its orientation is also changed during propagation. Thus, by applying a magnetic field a spin precession can be achieved.

In the previous section, we already pointed out that the superposition state $\psi_{j, \|}$, with equal total angular momentum but opposite spin orientation, results in an oscillation of the average spin along the cylinder axis. In Figs. 7(a) and 7(b) these oscillations of $\left\langle\sigma_{z}\right\rangle_{\|}$are shown for different values of $j$ at $B=0.13 \mathrm{~T}$. One finds that for higher total angular momentum values, the oscillation period is shorter, owing to the larger difference in Fermi wave vectors. In Fig. 5 the states 

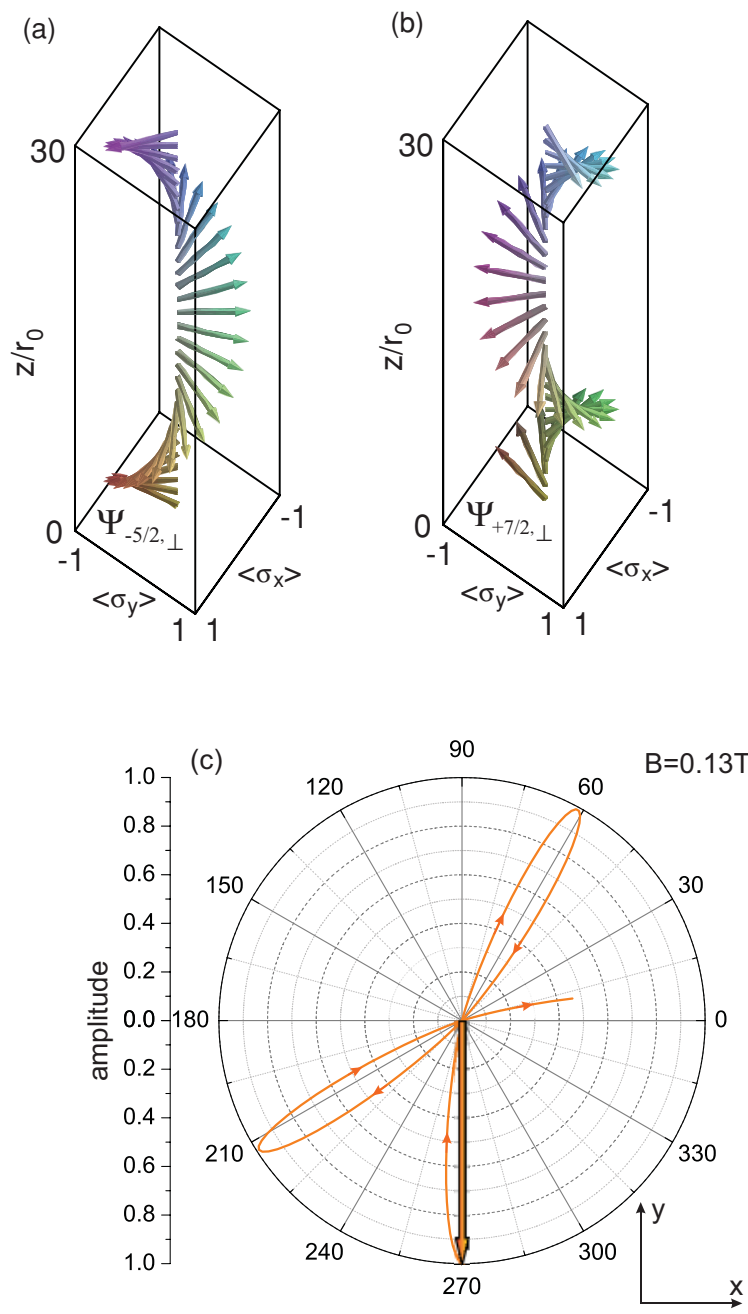

FIG. 6. (Color online) (a) Spin precession of electrons at the Fermi energy propagating along the wire axis for the superposition state $\psi_{-5 / 2, \perp}$ at $B=0.13 \mathrm{~T}$. (b) Corresponding spin precession for the state $\psi_{+7 / 2, \perp}$. (c) Spin orientation and magnitude of the sum of the contributions shown in (a) and (b) for a propagation from $z / r_{0}=0$ to 30 . The arrow indicates the direction of the initially injected spin.

contributing to $\psi_{+5 / 2, \|}$ are represented by triangles. Compared to the previously discussed $\psi_{j, \perp}$ states, here the difference in the Fermi vectors is relatively large, leading to a faster oscillation compared to the spin precession period shown in Fig. 6(b).

Once again, the application of an axial magnetic field breaks the symmetry of the $\psi_{ \pm j, \|}$ states. As can be inferred from Fig. 7(c), a different oscillation period is found for the $\psi_{+5 / 2, \|}$ and $\psi_{-5 / 2, \|}$ states. Thus, when these states are combined, a beating in the oscillation of the average spin appears.

\section{CONCLUSIONS}

In the previous two sections we have learned that an injected spin is strongly modulated while propagating through a cylindrical nanowire. For a spin injection along the wire axis, for example, by a ferromagnetic electrode, the spin is carried by superposition states with equal total angular momenta. In analogy to the spin-field-effect transistor based on a planar $2 \mathrm{DEG},{ }^{10}$ a transistor structure can be realized by placing a
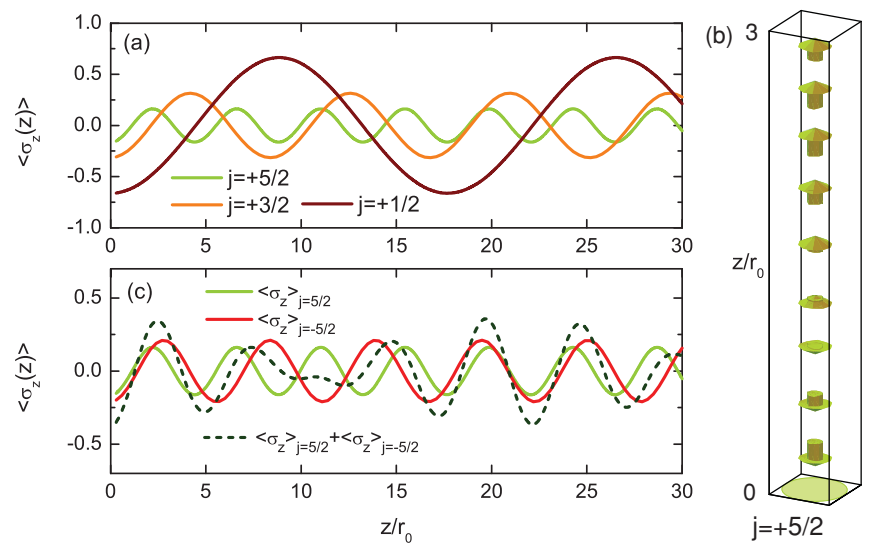

FIG. 7. (Color online) (a) Spin orientation $\left\langle\sigma_{z}\right\rangle_{\|}$along the wire axis for the superposition states $\psi_{j, \|}$ with $j=+1 / 2,+3 / 2$, and $+5 / 2$ at $B=0.13 \mathrm{~T}$. (b) Illustration of the oscillation of the average spin

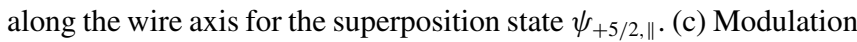
of the total spin orientation $\left\langle\sigma_{z}\right\rangle$ (dashed line) resulting from a combination of the $\psi_{-5 / 2, \|}$ and $\psi_{+5 / 2, \|}$ states at $B=0.13 \mathrm{~T}$.

second magnetic electrode at the opposite terminal of the nanowire as a spin detector. Control of the spin orientation can by achieved by manipulating the strength of the Rashba effect by means of a gate electrode. By applying a bias voltage to the gate, the strength of the electric field $\overrightarrow{\mathcal{E}}$ in the surface $2 \mathrm{DEG}$ is adjusted. To obtain uniform control within the channel, a so-called wrap-around gate should be preferred. ${ }^{36}$ Usually, in a realistic situation a larger number of states with different total angular momenta $j$ is occupied. As we observed, for each superposition state $\psi_{j, \|}$, different oscillation periods are found. This leads to a rather complex modulation of the spin along the axial direction. An obvious strategy for simplification is to reduce the number of occupied states, that is, by depleting the channel by means of a gate. Another possibility might be to occupy only certain states by means of $k$-selective filters. This might be realized by means of an injection through a single or a resonant tunneling barrier. As pointed out in Sec. III, one possible way to model this situation is to assume the formation of a state with a Gaussian distribution around the average momentum.

In addition to a spin injection and detection along the wire axis, it is also possible to inject spins in the transversal direction. Here, the spins are carried by superposition states $\psi_{j, \perp}$ constituted of states with different total angular momenta $j$. As long as no magnetic field is applied, the spin is exclusively modulated in the plane spanned by the injection orientation and the wire axis. Here, the output signal in a spin-field-effect transistor is gained by gate-modulating the spin orientation along or opposite to a detector electrode, which is polarized parallel or antiparallel to the injector. By applying an axially oriented magnetic field, spin precession about the wire axis can be achieved. This additional feature might be an interesting option to implement more complex functionalities in spin electronic devices.

In conclusion, we have shown that semiconductor nanowires affected by Rashba spin-orbit coupling are promising candidates for future nanowire-based spin electronic devices. The complex spin dynamics in these cylindrically 
shaped conductors provides many opportunities to tailor the device functionality.

\section{ACKNOWLEDGMENTS}

We thank N. Demarina (Forschungszentrum Jülich) for fruitful discussions regarding the Schrödinger-Poisson solver in cylindrical systems and U. Zülicke (Massey University, New Zealand) and R. Winkler (Northern Illinois University, USA) for discussions on the Rashba effect at semiconductor interfaces. Furthermore, we acknowledge the support of or work by S. Blügel and D. Grützmacher (Forschungszentrum Jülich). This work was supported by the Deutsche Forschungsgemeinschaft through Grant No. FOR 912.
*Corresponding author; th.schaepers@fz-juelich.de

${ }^{1}$ C. Thelander, P. Agarwal, S. Brongersma, J. Eymery, L. Feiner,

A. Forchel, M. Scheffler, W. Riess, B. Ohlsson, U. Gösele, and L. Samuelson, Materials Today 9, 28 (2006).

${ }^{2}$ W. Lu and C. M. Lieber, J. Phys. D: Appl. Phys. 39, R387 (2006).

${ }^{3}$ H. Lüth, Solid Surfaces, Interfaces and Thin Films, 5th ed. (Springer-Verlag, Berlin, Heidelberg, New York, 2010).

${ }^{4}$ Y. Tserkovnyak and B. I. Halperin, Phys. Rev. B 74, 245327 (2006).

${ }^{5}$ T. Richter, Ch. Blömers, H. Lüth, R. Calarco, M. Indlekofer, M. Marso, and Th. Schäpers, Nano Lett. 8, 2834 (2008).

${ }^{6}$ G. Ferrari and G. Cuoghi, Phys. Rev. Lett. 100, 230403 (2008).

${ }^{7}$ G. Ferrari, A. Bertoni, G. Goldoni, and E. Molinari, Phys. Rev. B 78, 115326 (2008).

${ }^{8}$ L. I. Magarill, D. A. Romanov, and A. V. Chaplik, JETP Lett. 64, 460 (1996).

${ }^{9}$ L. I. Magarill, D. A. Romanov, and A. V. Chaplik, J. Exp. Theor. Phys. 86, 771 (1998).

${ }^{10}$ S. Datta and B. Das, Appl. Phys. Lett. 56, 665 (1990).

${ }^{11}$ J. C. Egues, G. Burkard, and D. Loss, Appl. Phys. Lett. 82, 2658 (2003).

${ }^{12}$ J. Schliemann, J. C. Egues, and D. Loss, Phys. Rev. Lett. 90, 146801 (2003).

${ }^{13}$ J. Nitta, T. Akazaki, H. Takayanagi, and T. Enoki, Phys. Rev. Lett. 78, 1335 (1997).

${ }^{14}$ G. Engels, J. Lange, Th. Schäpers, and H. Lüth, Phys. Rev. B 55, R1958 (1997).

15 Th. Schäpers, G. Engels, J. Lange, Th. Klocke, M. Hollfelder, and H. Lüth, J. Appl. Phys. 83, 4324 (1998).

${ }^{16}$ Y. Bychkov and E. I. Rashba, J. Phys. C 17, 6039 (1984).

${ }^{17}$ J. Nitta, F. E. Meijer, and H. Takayanagi, Appl. Phys. Lett. 75, 695 (1999).

${ }^{18}$ A. A. Kiselev and K. W. Kim, Appl. Phys. Lett. 78, 775 (2001).

${ }^{19}$ U. Zülicke and M. Governale, Phys. Rev. B 65, 205304 (2002).

${ }^{20}$ V. A. Guzenko, J. Knobbe, H. Hardtdegen, Th. Schäpers, and A. Bringer, Appl. Phys. Lett. 88, 032102 (2006).
${ }^{21}$ V. A. Guzenko, A. Bringer, J. Knobbe, H. Hardtdegen, and Th. Schäpers, Appl. Phys. A 87, 577 (2007).

${ }^{22}$ S. Jin, J. Waugh, T. Matsuura, S. Faniel, H. Wu, and T. Koga, Physics Procedia 3, 1321 (2010).

${ }^{23}$ O. Entin-Wohlman, A. Aharony, Y. Tokura, and Y. Avishai, Phys. Rev. B 81, 075439 (2010).

${ }^{24}$ M. Trushin and J. Schliemann, Physica E 40, 1446 (2008).

${ }^{25}$ P. Wenk and S. Kettemann, Phys. Rev. B 81, 125309 (2010).

${ }^{26}$ G. Petersen, S. Estévez Hernández, R. Calarco, N. Demarina, and Th. Schäpers, Phys. Rev. B 80, 125321 (2009).

${ }^{27}$ A. E. Hansen, M. T. Björk, I. C. Fasth, C. Thelander, and L. Samuelson, Phys. Rev. B 71, 205328 (2005).

${ }^{28}$ S. Dhara, H. S. Solanki, V. Singh, A. Narayanan, P. Chaudhari, M. Gokhale, A. Bhattacharya, and M. M. Deshmukh, Phys. Rev. B 79, 121311 (2009)

${ }^{29}$ P. Roulleau, T. Choi, S. Riedi, T. Heinzel, I. Shorubalko, T. Ihn, and K. Ensslin, Phys. Rev. B 81, 155449 (2010).

${ }^{30}$ S. Estévez Hernández, M. Akabori, K. Sladek, Ch. Volk, S. Alagha, H. Hardtdegen, M. G. Pala, N. Demarina, D. Grützmacher, and Th. Schäpers, Phys. Rev. B 82, 235303 (2010).

${ }^{31}$ S. Lamari, Phys. Rev. B 67, 165329 (2003).

${ }^{32}$ C. Schierholz, T. Matsuyama, U. Merkt, and G. Meier, Phys. Rev. B 70, 233311 (2004).

${ }^{33}$ K. Smit, L. Koenders, and W. Mönch, J. Vac. Sci. Technol. B 7, 888 (1989).

${ }^{34} \mathrm{R}$. Winkler, Spin-Orbit Coupling Effects in Two-dimensional Electron and Hole Systems, Springer Tracts in Modern Physics (Springer-Verlag, New York, 2003), Vol. 191.

${ }^{35} \mathrm{We}$ are aware that by our approach of assuming an infinite barrier at the wire boundary the leakage of the wave function into the barrier is neglected. It has been shown that the leakage of the wave function can contribute significantly to the Rashba effect. ${ }^{15,34}$ However, since the InAs interface to the environment, for example, to vacuum, cannot be described with sufficient accuracy, we decided to assumed a barrier of infinite height and adjusted the strength of spin-orbit coupling to the experimental data. ${ }^{32}$

${ }^{36}$ T. Bryllert, L.-E. Wernersson, T. Lowgren, and L. Samuelson, Nanotechnol. 17, S227 (2006). 